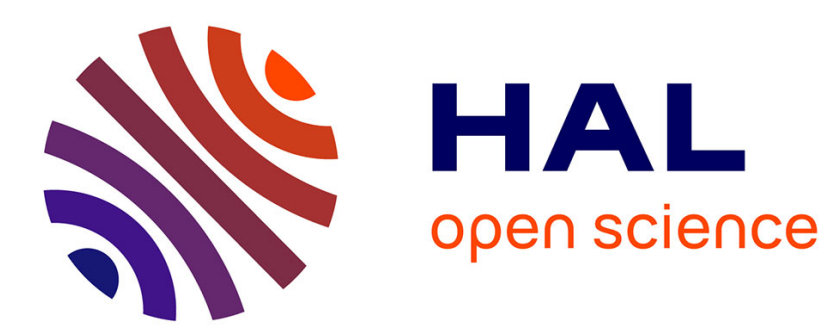

\title{
Using nonlinear normal modes to analyze forced vibrations
}

\author{
Konstantin Avramov
}

\section{To cite this version:}

Konstantin Avramov. Using nonlinear normal modes to analyze forced vibrations. International Applied Mechanics, 2008, 10.1007/s10778-009-0153-3 . hal-01338618

\section{HAL Id: hal-01338618 https://hal.science/hal-01338618}

Submitted on 28 Jun 2016

HAL is a multi-disciplinary open access archive for the deposit and dissemination of scientific research documents, whether they are published or not. The documents may come from teaching and research institutions in France or abroad, or from public or private research centers.
L'archive ouverte pluridisciplinaire HAL, est destinée au dépôt et à la diffusion de documents scientifiques de niveau recherche, publiés ou non, émanant des établissements d'enseignement et de recherche français ou étrangers, des laboratoires publics ou privés. 


\title{
USING NONLINEAR NORMAL MODES TO ANALYZE FORCED VIBRATIONS
}

\author{
K. V. Avramov
}

\begin{abstract}
The paper proposes a method to analyze forced vibrations in nonlinear systems. The procedure combines Rauscher's method and Pierre-Shaw nonlinear modes. Results from an analysis of the forced vibrations of a shallow arch are presented as an example
\end{abstract}

Keywords: nonlinear forced vibrations, discrete systems, Rauscher's method, Pierre-Shaw nonlinear modes, shallow arch

Introduction. There are many methods to analyze the forced vibrations of nonlinear discrete systems. The mechanics of continuum systems uses the multiple-scales methods, Van der Pol's method, Mel'nikov's method [3, 15, 18, 22]. The forced vibrations of essentially nonlinear systems are studied using the harmonic-balance method and the parameter continuation algorithm [1, 4, 21].

Rauscher's method was proposed in [16] to analyze nonautonomous systems with one degree of freedom. Denote the generalized coordinate of the system by $q$. We first solve an independent problem $q(t)$. Its solution is inverted and represented as $t=t(q)$. This function is substituted into a nonautonomous system, and this system becomes pseudoautonomous. Rauscher's method is generalized in the monograph [6]. In [2], Chebyshev polynomials are used to obtain the function $t=t(q)$. That the function $t=t(q)$ exists for a wide class of dynamic systems was proved in [17]. Rauscher's method was used in [17] for a qualitative analysis of dynamic systems with one degree of freedom. In [4], Kauderer-Rosenberg nonlinear normal modes are combined with Rauscher's method to analyze discrete mechanical systems with an arbitrary number of degrees of freedom. Forced vibrations close to a rectilinear normal mode of a mechanical system with two degrees of freedom are studied in [21]. Rauscher's method is used in [10] to analyze a mechanical system consisting of a linear subsystem and a Mises truss.

Note that all the above-cited publications use Rauscher's method together with Kauderer-Rosenberg nonlinear normal modes, which represent motions in the configuration space.

Nonlinear normal modes are an effective tool for solving complicated engineering problems. They were used in [5, 7] to analyze the possibility of suppressing mechanical vibrations and in [11] for studying nonlinear vibrations of rotating twisted rods. The studies $[7,8,12,13]$ are concerned with the vibrations of cylindrical shells and polygonal plates.

The present paper proposes a new method to analyze forced vibrations. The method combines Rauscher's method and nonlinear normal modes, which are two-dimensional invariant manifolds. Such normal modes were proposed in [19, 20]. The method can be used to analyze engineering systems with a great number of degrees of freedom.

1. Combination of Nonlinear Normal Modes and Rauscher's Method. Consider a discrete system

$$
\ddot{q}_{i}+\omega_{i}^{2} q_{i}+R_{i}\left(q_{1}, \ldots, q_{m}\right)=h_{i} \cos (\Omega t), \quad i=\overline{1, m}
$$

undergong forced vibrations.

Assume that the vibrations are not damped. The vector function $R_{i}\left(q_{1}, \ldots, q_{m}\right)$ describes nonlinear terms that contain the generalized coordinates raised to second, third, and other powers. It is assumed that the natural frequencies $\omega_{i}$ do not satisfy the internal-resonance condition. 
Assume that the excitation frequency $\Omega$ is close to the natural frequency $\omega_{l}$. Since there is no internal resonance, we can assume that the amplitude of the generalized coordinate $q_{l}$ is much higher than all the other generalized coordinates in the neighborhood of the principal resonance. For the purpose of vibration analysis, we will use an iterative procedure. At the first iteration, we set $q_{l} \neq 0$ and $q_{\mu}=0, \mu=\overline{1, m}, \mu \neq l$. Then an oscillator with one degree of freedom follows from system (1):

$$
\ddot{q}_{l}+\omega_{l}^{2} q_{l}+\widetilde{R}_{l}\left(q_{l}\right)=h_{l} \cos (\Omega t)
$$

where $\widetilde{R}_{l}\left(q_{l}\right)=R_{l}\left(0, \ldots, 0, q_{l}, 0, \ldots, 0\right)$.

Following Rauscher's method, we represent the solution of system (2) as

$$
\cos \Omega t=r\left(q_{l}\right)=\alpha_{0}+\alpha_{1} q_{l}+\alpha_{2} q_{l}^{2}+\ldots
$$

The idea of combining nonlinear normal modes and Rauscher's method is as follows. Substituting (3) into system (1), we obtain a so-called pseudoautonomous dynamic system [10,21]. Then nonlinear normal modes are analyzed in the pseudoautonomous dynamic system [19]. Using nonlinear normal modes, we reduce the system of $m$ equations (1) to one equation analyzed approximately.

Let us now obtain the solution of Eq. (2) in the form (3). To this end, we apply the harmonic balance method to system (2). The solution is represented as

$$
q_{l}=A_{0}+A_{1} \cos \Omega t+A_{2} \cos 2 \Omega t+A_{3} \cos 3 \Omega t+\ldots
$$

Restricting the analysis to three Fourier harmonics, we substitute solution (4) into (2) and balance the harmonics. Doing so gives a system of four nonlinear algebraic equations for $\left(\Omega, A_{0}, A_{1}, A_{2}, A_{3}\right)$. Its general form is

$$
\Phi_{v}\left(\Omega, A_{0}, A_{1}, A_{2}, A_{3}\right)=0, v=\overline{1,4} .
$$

The objective of the analysis is to plot the amplitude-frequency characteristic at the principal resonance $\Omega \approx \omega_{l}$. This is why the value of $A_{1}$ is incremented, and the system of the nonlinear algebraic equations (5) is solved for each value of $A_{1}$. As a result, we obtain parameters $\left(\Omega, A_{0}, A_{2}, A_{3}\right)$. Then, the values of $\Omega, A_{0}, A_{2}$, and $A_{3}$ are used to determine the coefficients of series (3): $\alpha_{0}, \alpha_{1}, \alpha_{2}, \ldots$

Let us outline a procedure of determining these coefficients. To this end, we represent the solution of Eq. (4) as

$$
q_{l}=A_{0}-A_{2}+z\left(A_{1}-3 A_{3}\right)+z^{2} 2 A_{2}+z^{3} 4 A_{3}+\ldots, \quad z=\cos \Omega t .
$$

Next, series (3) is substituted into (6) and the coefficients of like powers of $q_{l}$ are equated. As a result, we obtain a cubic equation for $\alpha_{0}$ :

$$
4 A_{3} \alpha_{0}^{3}+2 A_{2} \alpha_{0}^{2}+\left(A_{1}-3 A_{3}\right) \alpha_{0}+A_{0}-A_{2}=0 .
$$

The parameters $\alpha_{1}, \alpha_{2}$, and $\alpha_{3}$ are defined by

$$
\begin{gathered}
\alpha_{1}=\frac{1}{A_{1}-3 A_{3}+4 A_{2} \alpha_{0}+12 A_{3} \alpha_{0}^{2}}, \\
\alpha_{2}=-\frac{\alpha_{1}^{2}\left(12 A_{3} \alpha_{0}+2 A_{2}\right)}{A_{1}-3 A_{3}+4 A_{2} \alpha_{0}+12 A_{3} \alpha_{0}^{2}}, \\
\alpha_{3}=-\frac{4 \alpha_{1}\left(A_{2} \alpha_{2}+A_{3} \alpha_{1}^{2}+6 A_{3} \alpha_{0} \alpha_{2}\right)}{A_{1}-3 A_{3}+4 A_{2} \alpha_{0}+12 A_{3} \alpha_{0}^{2}} .
\end{gathered}
$$

Equation (3) is substituted into the nonautonomous dynamic system (2) to obtain a pseudoautonomous dynamic system: 


$$
\ddot{q}_{i}+\omega_{i}^{2} q_{i}+R_{i}\left(q_{1}, \ldots, q_{m}\right)=h_{i}\left(\alpha_{0}+\alpha_{1} q_{l}+\alpha_{2} q_{l}^{2}+\ldots\right), \quad i=\overline{1, m}
$$

For the purpose of further analysis, we change variables:

$$
q_{i}=q_{0, i}+\eta_{i}(t)
$$

where $q_{0, i}(i=\overline{1, m})$ are the coordinates of the fixed point of dynamic system (9) that are determined from a system of $m$ nonlinear algebraic equations:

$$
\omega_{i}^{2} q_{0, i}+R_{i}\left(q_{0,1}, \ldots, q_{0, m}\right)=h_{i}\left(\alpha_{0}+\alpha_{1} q_{0, l}+\alpha_{2} q_{0, l}^{2}+\ldots\right), i=\overline{1, m}
$$

The dynamic system (9) takes the following form for the variables $\eta_{1}, \ldots, \eta_{m}$ :

$$
\ddot{\eta}_{i}+\sum_{j=1}^{m} \alpha_{i j} \eta_{j}+F_{i}\left(\eta_{1}, \ldots, \eta_{m}\right)=0, \quad i=\overline{1, m},
$$

where $F_{i}\left(\eta_{1}, \ldots, \eta_{m}\right)$ are nonlinear functions.

The matrix $\Lambda=|| \alpha_{i j} \|_{j=1, \overline{1, m}}^{i=\overline{1, m}}$ is represented as

$$
\Lambda=U A U^{-1}
$$

where $U$ is a matrix consisting of the eigenvectors of the matrix $\Lambda ; A=\operatorname{diag}\left(v_{1}^{2}, \ldots, v_{m}^{2}\right)$. We introduce new variables:

$$
\xi=U^{-1} \eta, \quad\left(\xi=\left[\xi_{1}, \ldots, \xi_{m}\right], \quad \eta=\left[\eta_{1}, \ldots, \eta_{m}\right]^{\mathrm{T}}\right) .
$$

Then the dynamic system (12) takes the form

$$
\ddot{\xi}_{i}+v_{i}^{2} \xi_{i}+L_{i}\left(\xi_{1}, \ldots, \xi_{m}\right)=0, i=\overline{1, m}
$$

where $L_{i}\left(\xi_{1}, \ldots, \xi_{m}\right), i=\overline{1, m}$, is the nonlinear part of the dynamic system.

To analyze the dynamic system (15), we will use Pierre-Shaw nonlinear normal modes [19]. Then, all the generalized coordinates $\xi_{i}$ and velocities $v_{i}=\dot{\xi}_{i}$ are expressed in terms of one coordinate $\xi_{l}$ and velocity $v_{l}$ :

$$
\begin{gathered}
\xi_{k}=\xi_{k}\left(\xi_{l}, v_{l}\right)=F_{1}^{(1)} \xi_{l}^{2}+F_{2}^{(1)} \xi_{l} v_{l}+F_{3}^{(1)} v_{l}^{2}+\ldots, \\
v_{k}=v_{k}\left(\xi_{l}, v_{l}\right)=F_{1}^{(2)} \xi_{l}^{2}+F_{2}^{(2)} \xi_{l} v_{l}+F_{3}^{(2)} v_{l}^{2}+\ldots, \\
k=\overline{1, m}, \quad k \neq l,
\end{gathered}
$$

where $F_{1}^{(1)}, F_{2}^{(1)}, \ldots$ are unknown coefficients to be determined.

Let us differentiate Eq. (16) with respect to time and use Eq. (15). Doing so gives the following system of partial differential equations:

$$
\begin{gathered}
v_{k}\left(\xi_{l}, v_{l}\right)=\frac{\partial \xi_{k}}{\partial \xi_{l}} v_{l}-\frac{\partial \xi_{k}}{\partial v_{l}}\left\{v_{l}^{2} \xi_{l}+L_{l}\left[\xi_{1}\left(\xi_{l}, v_{l}\right), \ldots, \xi_{l}, \ldots, \xi_{m}\left(\xi_{l}, v_{l}\right)\right]\right\}, \\
v_{k}^{2} \xi_{k}\left(\xi_{l}, v_{l}\right)+L_{k}\left[\xi_{1}\left(\xi_{l}, v_{l}\right), \ldots, \xi_{l}, \ldots, \xi_{m}\left(\xi_{l}, v_{l}\right)\right] \\
=-\frac{\partial v_{k}}{\partial \xi_{l}} v_{l}+\frac{\partial v_{k}}{\partial v_{l}}\left\{v_{l}^{2} \xi_{l}+L_{l}\left[\xi_{1}\left(\xi_{l}, v_{l}\right), \ldots, \xi_{l}, \ldots, \xi_{m}\left(\xi_{l}, v_{l}\right)\right]\right\}, \quad k=\overline{1, m}, k \neq l .
\end{gathered}
$$




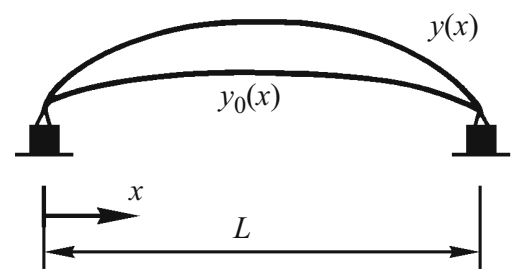

Fig. 1

Following [13], we substitute Eqs. (16) into (17) and collect the coefficients of like terms $\xi_{l}^{j_{1}} v_{l}^{j_{2}}, j_{1}=0,1,2, \ldots$, $j_{2}=0,1,2, \ldots$. As a result, we obtain a system of linear algebraic equations for $F_{1}^{(1)}, F_{2}^{(1)}, \ldots$. Solving this system yields functions (16).

Using Eq. (14), we write functions (16) for the variables $\eta_{1}, \ldots, \eta_{m}$ as

$$
\begin{gathered}
\eta_{k}=\eta_{k}\left(\eta_{l}, s_{l}\right)=C_{1}^{(1)} \eta_{l}+C_{2}^{(1)} s_{l}+C_{3}^{(1)} \eta_{l}^{2}+C_{4}^{(1)} \eta_{l} s_{l}+C_{5}^{(1)} s_{l}^{2}+\ldots, \\
s_{k}=s_{k}\left(\eta_{l}, s_{l}\right)=C_{1}^{(2)} \eta_{l}+C_{2}^{(2)} s_{l}+C_{3}^{(2)} \eta_{l}^{2}+C_{4}^{(2)} \eta_{l} s_{l}+C_{5}^{(2)} s_{l}^{2}+\ldots,
\end{gathered}
$$

where $s_{k}=\dot{\eta}_{k}, C_{1}^{(1)}, C_{2}^{(1)}, \ldots$ are known parameters.

We substitute Eq. (18) into (10) and the resulting functions into the equation $l$ of system (1). Doing so gives a dynamic system with one degree of freedom, which can be represented in the form (2). To solve this equation, we use the harmonic-balance method. The solution is presented in the form (4). Then, we obtain a system of four nonlinear algebraic equations for $\Omega, A_{0}, A_{2}$, and $A_{3}$. In the general case, this system can be represented in the form (5).

Let us now generalize the iterative procedure outlined above. At the first iteration, one equation (2) is solved and the contribution of the coordinates $q_{1}, q_{2}, \ldots, q_{l-1}, q_{l+1}, \ldots, q_{m}$ is neglected. At the second iteration, the nonlinear vibration mode (18) is used to take into account the contribution of all the coordinates to the dynamics of the system. The third, fourth, etc. iterations are set up similarly. If the values of $\Omega, A_{0}, A_{2}$, and $A_{3}$ obtained in two successive iterations are close, the iterative process is terminated. Then new value of $A_{1}$ is specified and a new point of the amplitude-frequency characteristic starts to be calculated.

2. Nonlinear Vibrations of a Shallow Arch. The above method can be used to analyze the nonlinear vibrations of a shallow arch (Fig. 1). The nonlinear dynamics of the arch is described by a system of integro-partial differential equations [14]:

$$
\begin{gathered}
A \rho y_{t t}+E I\left(y-y_{0}\right)_{x x x x}-H y_{x x}=\oint\left(x-\frac{L}{n}\right) F \cos (\Omega t), \\
H=\frac{E A}{2 L} \int_{0}^{L}\left(y_{x}^{2}-y_{0, x}^{2}\right) d x,
\end{gathered}
$$

where $A$ and $I$ are the cross-sectional area and moment of inertia; $y(x, t)$ is the current deflection of the arch; $E$ and $\rho$ are Young's modulus and density of the material; $y_{0}(x)$ is the initial deflection of the $\operatorname{arch} ; \delta(x-L / n)$ is the delta-function; $n$ is an arbitrary number; and $F \cos (\Omega t)$ is a concentrated force. We introduce the following dimensionless variables and parameters:

$$
x^{*}=\frac{x}{L}, \quad y^{*}=\frac{y}{r}, \quad t^{*}=t \sqrt{\frac{E I}{\rho A l^{4}}}, \quad y_{0}=r y_{0}^{*}, \quad \delta^{*}\left(x^{*}-\frac{1}{n}\right)=L \delta\left(x-\frac{L}{n}\right), \quad f=\frac{F L^{3}}{E I r},
$$

where $r$ is the cross-sectional radius of inertia.

Omittng the asterisks in (20), we represent the dynamic system (19) in the form 


$$
y_{t t}+\left(y-y_{0}\right)_{x x x x}-\frac{y_{x x}}{2} \int_{0}^{1}\left(y_{x}^{2}-y_{0, x}^{2}\right) d x=\oint\left(x-\frac{1}{n}\right) f \cos (\Omega t) .
$$

The initial deflection of the arch can be represented as

$$
y_{0}=\lambda_{1} \sin (\pi x)+\lambda_{2} \sin (2 \pi x)
$$

Then the vibrations of the arch can be described by

$$
y(x, t)=\left(\lambda_{1}+q_{1}(t)\right) \sin (\pi x)+\left(\lambda_{2}+q_{2}(t)\right) \sin (2 \pi x) .
$$

Next we set $n=2$, substitute Eqs. (22) and (23) into (21), and apply the Bubnov-Galerkin method. Doing so gives the following dynamic system:

$$
\begin{gathered}
\ddot{q}_{1}+\pi^{4} q_{1}+\frac{\pi^{4}}{4}\left(q_{1}+\lambda_{1}\right) \mu\left(q_{1}, q_{2}\right)=2 f \cos (\Omega t), \\
\ddot{q}_{2}+16 \pi^{4} q_{2}+\pi^{4}\left(q_{2}+\lambda_{2}\right) \mu\left(q_{1}, q_{2}\right)=0, \\
\mu\left(q_{1}, q_{2}\right)=q_{1}\left(q_{1}+2 \lambda_{1}\right)+4 q_{2}\left(q_{2}+2 \lambda_{2}\right) .
\end{gathered}
$$

Now we introduce normal coordinates $\left(x_{1}, x_{2}\right)$ of the linear part of system (24), which are determined from the linear relations

$$
\left[\begin{array}{l}
q_{1} \\
q_{2}
\end{array}\right]=\left[\begin{array}{rr}
r_{1} & r_{2} \\
1 & 1
\end{array}\right]\left[\begin{array}{l}
x_{1} \\
x_{2}
\end{array}\right]
$$

where

$$
\begin{gathered}
r_{j}=\frac{p_{j}^{2}-8 \lambda_{2}^{2}-16}{2 \lambda_{1} \lambda_{2}}, \quad j=1,2, \\
2 p_{1,2}^{2}=17+0.5 \lambda_{1}^{2}+8 \lambda_{2}^{2} \mp\left[225-15 \lambda_{1}^{2}+240 \lambda_{2}^{2}+0.25 \lambda_{1}^{4}+64 \lambda_{2}^{4}+8 \lambda_{1}^{2} \lambda_{2}^{2}\right]^{1 / 2} .
\end{gathered}
$$

The dynamic system (24) takes the following form for the variables $\left(x_{1}, x_{2}\right)$ :

$$
\ddot{x}_{i}+p_{i}^{2} x_{i}=\sigma\left[\theta_{i} f_{1} \cos \left(\Omega_{1} t\right)+A_{1}^{(i)} x_{1}^{2}+A_{2}^{(i)} x_{2}^{2}+A_{3}^{(i)} x_{1} x_{2}+A_{4}^{(i)} x_{1}^{3}+A_{5}^{(i)} x_{1}^{2} x_{2}+A_{6}^{(i)} x_{1} x_{2}^{2}+A_{7}^{(i)} x_{2}^{3}\right], i=1,2
$$

where $\theta_{1}=1, \theta_{2}=-1, \sigma=\frac{2 \lambda_{1} \lambda_{2}}{p_{1}^{2}-p_{2}^{2}}$, and $f_{1}=\frac{2 f}{\pi^{4}}$. The parameters $A_{1}^{(1)}, A_{2}^{(1)}, \ldots$ are omitted for brevity.

Let us analyze forced vibrations at the principal resonance. In this case, the excitation frequency $\Omega$ is close to $p_{1}$. We set $x_{2}=0$ at the first iteration. Then we obtain the following dynamic system:

$$
\ddot{x}_{1}+p_{1}^{2} x_{1}=\sigma\left[f_{1} \cos \left(\Omega_{1} t\right)+A_{1}^{(1)} x_{1}^{2}+A_{4}^{(1)} x_{1}^{3}\right] .
$$

The solution of this equation can be represented in the form (4). Applying the harmonic-balance method, we arrive at a system of nonlinear algebraic equations, which can be written in the form (5). If system (5) has been solved numerically, the solution of Eq. (27) could be represented in the form (3). This solution is substituted into (26). As a result, we obtain a pseudoautonomous dynamic system:

$$
\ddot{x}_{i}+p_{i}^{2} x_{i}=\sigma\left[\theta_{i} f_{1} r\left(x_{1}\right)+A_{1}^{(i)} x_{1}^{2}+A_{2}^{(i)} x_{2}^{2}+A_{3}^{(i)} x_{1} x_{2}+A_{4}^{(i)} x_{1}^{3}+A_{5}^{(i)} x_{1}^{2} x_{2} A_{6}^{(i)} x_{1} x_{2}^{2}+A_{7}^{(i)} x_{2}^{3}\right], \quad i=1,2 .
$$

Let us change variables as in (10). For (28), we have 


$$
x_{i}=x_{0, i}+\eta_{i}(t), \quad i=1,2 .
$$

Then the parameters $x_{0,1}$ and $x_{0,2}$ can be found from a system of nonlinear algebraic equations in the form (11). System (28) can be represented for the variables $\left(\eta_{1}, \eta_{2}\right)$ as

$$
\begin{gathered}
\ddot{\eta}_{i}+p_{i}^{2} \eta_{i}=\sigma\left[c_{i 1} \eta_{1}+c_{i 2} \eta_{2}+P_{i}^{(2)}\left(\eta_{1}, \eta_{2}\right)+P_{i}^{(3)}\left(\eta_{1}, \eta_{2}\right)\right], \\
P_{i}^{(2)}\left(\eta_{1}, \eta_{2}\right)=B_{1}^{(i)} \eta_{1}^{2}+B_{2}^{(i)} \eta_{2}^{2}+B_{3}^{(i)} \eta_{2} \eta_{1}, \\
P_{i}^{(3)}\left(\eta_{1}, \eta_{2}\right)=B_{4}^{(i)} \eta_{1}^{3}+B_{5}^{(i)} \eta_{1}^{2} \eta_{2}+B_{6}^{(i)} \eta_{2}^{2} \eta_{1}+B_{7}^{(i)} \eta_{2}^{3}, \quad i=\overline{1,2}
\end{gathered}
$$

where the parameters $B_{1}^{(1)}, B_{2}^{(1)}, \ldots$ are omitted for brevity.

We rearrange the linear part of system (30) for the normal coordinates using the equation

$$
\left[\begin{array}{l}
\eta_{1} \\
\eta_{2}
\end{array}\right]=\left[\begin{array}{rr}
\varsigma_{1} & \varsigma_{2} \\
1 & 1
\end{array}\right]\left[\begin{array}{l}
\xi_{1} \\
\xi_{2}
\end{array}\right], \quad \varsigma_{i}=\frac{\sigma c_{12}}{p_{1}^{2}-v_{i}^{2}-\sigma c_{11}}, \quad i=1,2
$$

The frequencies $v_{1}$ and $v_{2}$ are found as the roots of the biquadratic equation

$$
v^{4}+v^{2} R_{0}+R_{1}=0
$$

where $R_{0}=\sigma\left(c_{22}+c_{11}\right)-p_{2}^{2}-p_{1}^{2}, R_{1}=p_{1}^{2} p_{2}^{2}-\sigma c_{22} p_{1}^{2}-\sigma c_{11} p_{2}^{2}+\sigma^{2}\left(c_{11} c_{22}-c_{12} c_{21}\right)$.

System (30) takes the following form for the variables $\left(\xi_{1}, \xi_{2}\right)$ :

$$
\begin{gathered}
\ddot{\xi}_{i}+v_{i}^{2} \xi_{i}=\alpha\left[R_{i}^{(2)}\left(\xi_{1}, \xi_{2}\right)+R_{i}^{(3)}\left(\xi_{1}, \xi_{2}\right)\right], \\
R_{i}^{(2)}=D_{1}^{(i)} \xi_{1}^{2}+D_{2}^{(i)} \xi_{2}^{2}+D_{3}^{(i)} \xi_{1} \xi_{2}, \\
R_{i}^{(3)}=D_{4}^{(i)} \xi_{1}^{3}+D_{5}^{(i)} \xi_{1}^{2} \xi_{2}+D_{6}^{(i)} \xi_{1} \xi_{2}^{2}+D_{7}^{(i)} \xi_{2}^{3},
\end{gathered}
$$

where the parameters $D_{1}^{(i)}, D_{2}^{(i)}, \ldots$ are omitted for brevity.

The nonlinear mode (invariant manifold) is represented in the form (16) when $k=2$ and $l=1$. Using the approach outlined in Sec. 1, we express the coefficients $F_{1}^{(1)}, F_{2}^{(1)}, \ldots, F_{3}^{(2)}$ as

$$
\begin{gathered}
F_{1}^{(1)}=\frac{\alpha D_{1}^{(2)}\left(2 v_{1}^{2}-v_{2}^{2}\right)}{v_{2}^{2}\left(4 v_{1}^{2}-v_{2}^{2}\right)}, \quad F_{2}^{(2)}=-\frac{2 \alpha D_{1}^{(2)}}{4 v_{1}^{2}-v_{2}^{2}}, \\
F_{3}^{(1)}=\frac{2 \alpha D_{1}^{(2)}}{v_{2}^{2}\left(4 v_{1}^{2}-v_{2}^{2}\right)}, \quad F_{2}^{(1)}=F_{3}^{(2)}=F_{1}^{(2)}=0 .
\end{gathered}
$$

The invariant manifolds are represented for the variables $\left(\eta_{1}, \eta_{2}, s_{1}, s_{2}\right)$ as

$$
\begin{gathered}
\eta_{2}=\varsigma_{1}^{-1} \eta_{1}+\delta_{*}\left(F_{1}^{(1)} \eta_{1}^{2}+F_{3}^{(1)} s_{1}^{2}\right)+\ldots, \\
s_{2}=\varsigma_{1}^{-1} s_{1}+F_{2}^{(2)} \delta * \eta_{1} s_{1}+\ldots,
\end{gathered}
$$

where $\delta_{*}=\frac{\left(1-\varsigma_{2} \varsigma_{1}^{-1}\right)^{2}}{\varsigma_{1}\left(\varsigma_{1}-\varsigma_{2}\right)}$.

The motion in a nonlinear normal mode is described by a nonlinear ordinary differential equation: 




Fig. 2

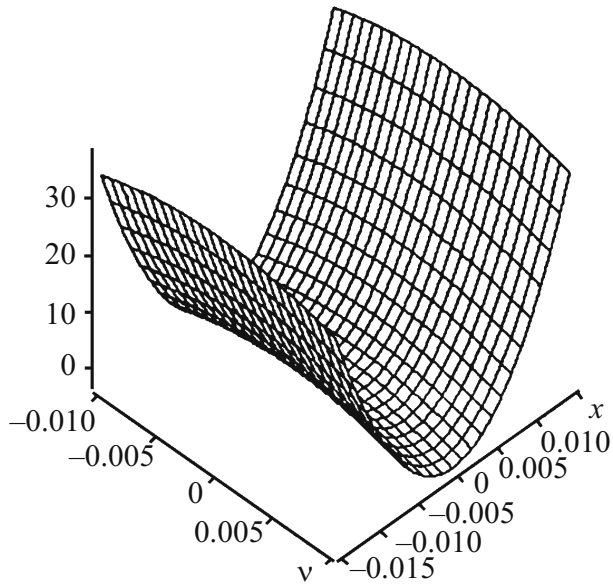

Fig. 3

$$
\ddot{x}_{1}+p_{1}^{2} x_{1}=\sigma\left[f_{1} \cos \Omega_{1} t+G_{0}^{(2)}+G_{1}^{(2)} x_{1}+G_{2}^{(2)} x_{1}^{2}+G_{3}^{(2)} \dot{x}_{1}^{2}+G_{4}^{(2)} x_{1}^{3}+G_{5}^{(2)} x_{1} \dot{x}_{1}^{2}\right]
$$

The parameters of system (36) are omitted for brevity. The periodic motions of system (36) are represented in the form (4). Then the parameters $\left(\Omega, A_{0}, A_{2}, A_{3}\right)$ follow from the system of nonlinear algebraic equations

$$
\widetilde{\Phi}_{v}\left(\Omega, A_{0}, A_{2}, A_{3}\right)=0, \quad v=\overline{1,4} .
$$

3. Numerical Vibration Analysis. Let us numerically analyze the forced vibrations of the shallow arch (19). We will use the following parameters of the system: $\lambda_{1}=5, \lambda_{2}=0.8, f=0.05$. Let the vibrations occur with a moderate amplitudes about the equilibrium state $q_{1}=q_{2}=0$. Snap-through buckling is not considered here. The natural frequencies of linear vibrations about the static equilibrium position are $p_{1}=2.9067$ and $p_{2}=5.1158$.

The procedure presented in Sec. 2 is used to analyze forced vibrations. The amplitude-frequency characteristic of forced vibrations (the modulus of the first Fourier harmonic $\left|A_{1}\right|$ versus the excitation frequency $\Omega_{1}$ ) is shown in Fig. 2. The dashed line represents the skeletal curve, while the solid line the curve of forced vibrations. The right branch of the amplitude-frequency characteristic (relative to the skeletal curve) is determined by the parameter $A_{1}>0$, and the left branch by $A_{1}<0$. The surface of the nonlinear normal mode for $A_{1}=-1.2$ is shown in Fig. 3 as an example.

Conclusions. The paper proposes an iterative procedure to analyze the forced vibrations of discrete nonlinear systems. This method combines Rauscher's method and nonlinear normal modes. Rauscher's method reduces a nonautonomous dynamic system to an iterative process of finding the normal mode in a pseudoautonomous system.

Nonlinear normal modes are an efficient approach to the analysis of dynamic systems with a great number of degrees of freedom $[11,20]$. Nonlinear normal modes can be applied to a dynamic system of high dimension resulting from finite-element discretization of an elastic medium.

\section{REFERENCES}

1. V. I. Gulyaev, V. A. Bazhenov, and S. L. Popov, Applied Problems in the Theory of Nonlinear Vibrations of Mechanical Systems [in Russian], Vyssh. Shk., Moscow (1989).

2. V. H. Kauderer, Nonlinear Mechanics [in German], Springer-Verlag, Berlin (1958).

3. V. D. Kubenko, P. S. Koval'chuk, and N. P. Podchasov, Nonlinear Vibrations of Cylindrical Shells [in Russian], Vishcha Shkola, Kyiv (1989).

4. L. I. Manevich, Yu. V. Mikhlin, and V. N. Pilipchuk, Method of Normal Vibrations for Essentially Nonlinear Systems [in Russian], Nauka, Moscow (1989). 
5. Yu. V. Mikhlin and S. N. Reshetnikova, "Dynamic analysis of a two-mass system with essentially nonlinear vibration damping," Int. Appl. Mech., 41, No. 1, 77-84 (2005).

6. J. J. Stocker, Nonlinear Oscillations in Mechanical and Electrical Systems, Interscience, New York (1950).

7. K. V. Avramov, "Nonlinear forced vibrations of a cylindrical shell with two internal resonances," Int. Appl. Mech., 42, No. 2, 169-175 (2006).

8. K. V. Avramov, "Chaotic frictional vibrations excited by a quasiperiodic load," Int. Appl. Mech., 42, No. 9, 1071-1076 (2006).

9. K. V. Avramov and Yu. V. Mikhlin, "Damping of free elastic vibrations in linear systems," Int. Appl. Mech., 41, No. 2, 203-209 (2005).

10. K. V. Avramov and Yu. V. Mikhlin, "Snap-through truss as an absorber of forced oscillations," J. Sound Vibr., 29, 705-722 (2006).

11. K. V. Avramov, C. Pierre, and N. V. Shyryaeva, "Nonlinear equations of flexural-flexural-torsional oscillations of rotating beams with arbitrary cross-section," Int. Appl. Mech., 44, No. 5, 582-590 (2008).

12. E. I. Bespalova, "Reaction of an anisotropic cylindrical shell to a moving load," Int. Appl. Mech., 43, No. 4, 425-431 (2007).

13. E. I. Bespalova, "Vibrations of polygonal plates with various boundary conditions," Int. Appl. Mech., 43, No. 5, 526-533 (2007).

14. C. S. Hsu, "Stability of shallow arches against snap-through under timewise step loads," ASME, J. Appl. Mech., 33, 31-39 (1968).

15. A. H. Nayfeh and D. T. Mook, Nonlinear Oscillations, John Wiley \& Sons, New York (1979).

16. M. Rauscher, "Steady oscillations of system with nonlinear and unsymmetrical elasticity," J. Appl. Mech., 5, A-169, 22-28 (1938).

17. R. M. Rosenberg, "Steady-state forced vibrations," Int. J. Nonlin. Mech., No. 1, 95-108 (1966).

18. J. A. Sanders and F. Verhulst, Averaging Methods in Nonlinear Dynamical Systems, Springer-Verlag, Berlin (1985).

19. S. W. Shaw and C. Pierre, "Normal modes for nonlinear vibratory systems," J. Sound Vibr., 164, 58-124 (1993).

20. S. W. Shaw, C. Pierre, and E. Pesheck, "Modal analysis-based reduced-order models for nonlinear structures- an invariant manifolds approach," The Shock Vibr. Digest, 31, 3-16 (1999).

21. A. F. Vakakis, L. I. Manevitch, Yu. V. Mikhlin, V. N. Pilipchuk, and A. A. Zevin, Normal Modes and Localization in Nonlinear Systems, Wiley Interscience, New York (1996).

22. S. Wiggins, Introduction to Applied Nonlinear Dynamical Systems and Chaos, Springer-Verlag, Berlin (1990). 